\title{
Maternal mood moderates the trajectory of emotional and behavioural problems from pre- to during the COVID-19 lockdown in preschool children
}

\author{
Alessandra Frigerio $^{1}$ (D) Francesca Nettuno ${ }^{1} \cdot$ Sarah Nazzari $^{1}$ (i) \\ Received: 6 May 2021 / Accepted: 30 November 2021 \\ (c) The Author(s), under exclusive licence to Springer-Verlag GmbH Germany 2021
}

\begin{abstract}
The COVID-19 outbreak and subsequent lockdown have dramatically impacted families' life, raising serious concerns about children's emotional wellbeing. However, few studies have investigated whether the impact of the COVID-19 lockdown on psychological adjustment in youngest can be moderated by maternal mood and, to our knowledge, none of them has adopted a longitudinal design. The main aim of the current study was to explore if the intensity and directionality of maternal mood symptoms moderated the trajectory of emotional and behavioural problems in Italian pre-schoolers from pre- to during the lockdown adopting a longitudinal design. To assess maternal anxiety and depression symptoms, the EPDS and the STAI-Y were filled in by 94 and 88 women before the lockdown, when their children were 1 (Wave P1) and 3 years old (Wave P2), respectively, and by 74 women during the lockdown, when their children were 4 years old (Wave L). Mothers also filled in the CBCL/1 $1 / 2-5$ to assess their children's emotional and behavioural problems at each assessment wave. As a whole, children's emotional and behavioural problems significantly increased from pre- to during the lockdown. Furthermore, maternal mood moderated this trajectory. In particular, greater maternal mood symptoms were significantly associated with a greater increase in emotional reactive, anxious-depressed, withdrawn and aggressive symptoms during the lockdown. These results contribute to shed light on the role played by maternal emotional wellbeing in buffering the impact of the COVID-19 lockdown on children's behavioural development. Albeit preliminary, the current findings highlight the need to provide timely psychological interventions to distressed mothers to help their children to better cope with the effects of the pandemic.
\end{abstract}

Keywords COVID-19 $\cdot$ Maternal anxiety $\cdot$ Maternal depression $\cdot$ Internalizing problems $\cdot$ Externalizing problems Preschool children

\section{Introduction}

The outbreak of the global health emergency related to the COVID-19 pandemic and subsequent lockdown had a profound impact on several domains of adults and children functioning, including their psychological wellbeing. A growing number of studies show the negative effects of the ongoing pandemic on adult mental health in samples of infected patients, patients with psychiatric disorders, health care workers and non-health care workers, reporting high

Alessandra Frigerio

alessandra.frigerio@lanostrafamiglia.it

1 Child Psychopathology Unit, Scientific Institute Eugenio Medea, Via Don Luigi Monza, 20, 23842 Bosisio Parini, LC, Italy levels of post-traumatic stress symptoms, as well as anxiety and depression symptoms in all groups (reviewed in [1]). However, less is known about mental health conditions in children [2], who have been exposed to significant changes in their daily routine linked to an extended lockdown. In particular, it is hypothesized that school closures, interruption of extra-scholastic activities, isolation from relatives and friends and exposition to increased family stress might have negatively affected children's mental health, leading to a greater risk of emotional and behavioural problems.

Beyond the risks associated with a greater exposition to internet and social media as well as to violence [3], abuse and neglect in their homes [4, 5], preliminary evidence shows several consequences of the lockdown on children's and adolescents' psychological wellbeing, mainly in terms of anxiety and depression symptoms but also irritability, inattention, boredom and fear of COVID-19 (for a review see 
$[6,7])$. Emerging literature on the impact of the COVID-19 emergency in Italy, which was the first European country to be heavily affected by the pandemic, provides conflicting findings. A few works showed an increase in both internalizing [8] and externalizing [9] problems during the national lockdown, although children's pre-pandemic functioning was assessed retrospectively. In contrast, two studies failed to detect any significant differences in children's psychological wellbeing during the lockdown, as compared to the Italian reference norms $[10,11]$.

Furthermore, only a handful of studies have investigated the impact of the COVID-19 lockdown on psychological functioning in preschool children, reporting increased irritability, inattention and clinging behaviour [12], sleep problems, poor appetite, agitation and separation-related anxiety $[8,13]$. Importantly, some studies also showed that preschool children would be at higher risk of displaying behavioural problems in comparison to older children, who can count on better coping strategies [14, 15]. In the light of these findings, scholars have tried to identify those family proximal factors that may exacerbate or, on the contrary, attenuate the impact of the COVID-19 containment measures on psychological functioning in preschool and school age children. Insofar as parents, especially mothers, were exposed to a heightened risk of experiencing mood symptoms and stress during the lockdown $[9,16]$, it is likely that some of them were less able to fulfil their parental role, with probable consequences on the psychological health of their children. Indeed, a large epidemiological study [14], carried out in China during the COVID-19 pandemic, shows that mental disorders in parents were more likely to be associated with greater children's psychosocial problems during the lockdown in a sample including 12,163 children aged 2-5 years. However, only few studies have investigated the impact of parental mood symptoms on children's behavioural problems during the lockdown, mainly focusing on COVID-19 pandemic-related anxiety, with conflicting results. Indeed, while Zreik et al. [17] found an association between acute COVID19 anxiety in mothers and sleep problems in their preschool age children, Crescentini et al. [7] reported that parents who had more fear of contagion attributed lower depression and somatic symptoms to their school age children. Furthermore, while there is robust evidence that maternal depression is significantly linked to socio-emotional development in preschool years [188], as far as we know, only Glynn and colleagues [19] recently reported the negative effects of maternal depression on both internalizing and externalizing symptoms in an at-risk sample of preschool children during the COVID-19 lockdown. Last, no studies have investigated the effects of both maternal anxiety and depression on children's behavioural problems during the lockdown, taking also into account that these disorders are often comorbid. In the current study, we examined comorbid anxiety and depression symptoms by distinguishing total symptom levels (i.e. symptom intensity) from whether mothers showed preponderance of anxiety vs depression symptoms (i.e. symptom directionality). Thus, in line with previous works [20, 21], we investigated overall symptom intensity as indexed by [(Anxiety + Depression)/2] and symptom directionality as indexed by [(Anxiety - Depression)/2].

Furthermore, despite the fact that previous investigations contributed to shed light on stress-related consequences of the COVID-19 lockdown on parents' and children's psychological wellbeing, evidence is limited by the cross-sectional design in which data related to children's pre-pandemic functioning are missing or based on retrospective reports [7]. To our knowledge, only two studies adopted a longitudinal design to investigate the impact of the lockdown on children's psychological well-being. Gassman-Pines et al. [22] assessed daily uncooperative behaviours and worry in a sample of 2-7 years old American children and showed no significant changes in the frequency of these behaviours immediately before the COVID-19 outbreak and during the lockdown. Cantiani et al. [23] compared emotional and behavioural problems pre- and during the lockdown, in 2-6 years old Italian children with typical development and at high familial risk for neurodevelopmental disorders, showing an increase of anxiety, depressive and externalizing symptoms during the lockdown regardless of the family risk. However, both studies did not take into account gender-related differences in children's behavioural development. Furthermore, the short time interval between the pre-lockdown and lockdown assessment in the former study and wide heterogeneity in time interval assessment between pre- and during the lockdown (ranging from 2 to 43 months) in the latter limit possible inferences about the impact of COVID-19 containment measures on children's developmental trajectory of behavioural problems.

The main aim of the current study was to investigate the impact of the COVID-19 lockdown on preschool children's psychological wellbeing in comparison to that preceding the pandemic, using specific time points, as well as to test the role played by the intensity and directionality (anxiety vs depression) of maternal mood symptoms in explaining individual differences in children's psychological adjustment. Specifically, the objectives of the study were:

a) to test if children showed an increase in emotional and behavioural problems during the lockdown compared to pre-pandemic assessments;

b) to explore if the intensity and directionality of maternal mood symptoms moderated the trajectory of emotional and behavioural problems in children from pre-pandemic to during the lockdown, controlling for some potential confounders (i.e. gender, intensity and directionality of maternal mood symptoms before the pandemic). As no 
previous studies have employed this approach in relation to this topic, no a priori hypotheses were formulated.

\section{Methods}

\section{Procedure and participants}

This study was designed and conducted in Lombardy between April 22 and June 3, 2020, during the lockdown restriction due to the COVID-19 pandemic ordered by the Italian Government on March 9. Although from May 3 to June 3 some restrictions (i.e. reopening of several working activities and possibility to do individual open air sports and activities) were reduced by the Government, most of them remained, including school closures, social activities and child confinement at home. At that time, Lombardy had the $37.8 \%$ of the total cases in Italy (Istituto Superiore di Sanità (ISS), Rome, 26 May 2020) and was characterized by a high spread of contagion within the provinces, with more than 150 cases per 100 thousand residents (Istat Report 4 June 2020).

Participants are part of an ongoing longitudinal study (EDI study: Effect of Depression on Infants) which aims at investigating the effects of antenatal maternal stress on several domains of child's development starting from birth [24-26]. For the current study, only emotional and behavioural data collected during the last two waves before the lockdown (Pre Lockdown waves, P1 and P2), when children were 12 months (mean $=13.74 ; \mathrm{SD}=1.63$ months) and 3 years (mean $=3.46 ; \mathrm{SD}=0.31$ years) of age, and during the lockdown (Lockdown wave, L), when children were 4 years of age (mean $=4.20 ; \mathrm{SD}=0.61$ years), were taken into account. During the P1 and P2 waves, mothers were asked to fill in questionnaires concerning their depressive (Edinburgh Postnatal Depression Scale, EPDS, [27]) and anxiety (State-Trait Anxiety Questionnaire Inventory-Y Form, STAI-Y, [28]) symptoms, as well as children's emotional and behavioural problems (Child Behavior Checklist, CBCL/1 1/2-5, [29]) during lab sessions at the IRCCS Eugenio Medea. During the $\mathrm{L}$ wave, mothers were invited to fill in the same questionnaires and an ad hoc form on the effects of COVID-19 through the REDCap electronic data capture tools hosted at IRCCSE. Medea [30] and the ASEBA Web platform.

All parents gave their written informed consent to each wave of the study. The experimental procedures were carried out in accordance with the ethical standards of the Declaration of Helsinki (BMJ 1991; 302: 1194). The study was approved by the Ethics Committee of the Scientific Institute E. Medea.

Depressive and anxiety symptoms were assessed by the EPDS and the STAI-Y, that were filled in by, respectively,
94, 88 and 74 mothers at waves P1, P2, L, while emotional and behavioural data, assessed by the CBCL/1 $1 / 2-5$, were available for $94($ Males $=50)$ at P1, $88($ Males $=47)$ at P2 and 59 (Males $=32$ ) participants at the $\mathrm{L}$ wave. Participants at the $\mathrm{L}$ wave did not differ from those who took part only in (one or both) the pre-lockdown waves on any demographic variables (i.e. child's gender $\left(\chi^{2}(1)=0.04 ; \mathrm{p}=0.56\right.$ ), maternal education $\left(\chi^{2}(1)=1.25 ; \mathrm{p}=0.22\right)$, paternal education $\left(\chi^{2}\right.$ $(1)=0.77 ; \mathrm{p}=0.26)$ and socioeconomic status $\left(\chi^{2}(2)=0.69\right.$; $\mathrm{p}=0.71$ ), assessed by the Hollingshead (1975) classification for parental occupation [31] or maternal mood symptoms (all $p \mathrm{~s}>0.05$ ).

\section{Measures}

\section{State-Trait Anxiety Inventory-Y Form (STAI-Y) [28]}

The STAI-Y is a 40 items self-rated questionnaire, which are rated on a 4-point Likert scale from 1 to 4 . Measures of non-chronic and chronic forms of anxiety can be obtained, respectively, through the State anxiety (STAI-S) and the Trait (STAI-T) subscales of the STAI-Y scale. Each subscale includes 20 items, with a total score ranging from 20 to 80 . For the purposes of this study, only the State Anxiety subscale was employed, as we were interested in investigating current maternal anxiety symptoms during the lockdown. The Italian version of the STAI-Y shows good psychometrics properties [32]. Repeated Measures ANOVA showed a statistically significant effect of time on the STAI-S ( $F$ $(2,128)=29.06 ; p<0.001)$ score. Post hoc test revealed that scores at the L wave were significantly higher than those obtained in the previous waves (both $p \mathrm{~s}<0.001$ ).

\section{Edinburgh Postnatal Depression Scale (EPDS) [27]}

The EPDS is a widely employed self-rated questionnaire to assess the presence and severity of the depressive symptoms in the past week. It consists of 10 items which are rated on a 4-point Likert scale from 0 to 3 . The Italian version of the EPDS employed in this study has shown good psychometric properties [33]. Repeated Measures ANOVA showed a statistically significant effect of time on the EPDS $(F(2,132)=8.85 ; p<0.001)$ score. Post hoc test revealed that scores at the $\mathrm{L}$ wave were significantly higher than those obtained in the previous waves (both $p \mathrm{~s}<0.05$ ).

\section{Child Behavior Checklist (CBCL/1 1/2-5) [28]}

The CBCL/1 $1 / 2-5$ is a widely employed questionnaire to assess behavioural and emotional problems in children between 1.5 and 5 years of age. It is composed by 99 items which are rated on a 3-point scale (0: not true, 1: partly true or 2 : very true, often true). The CBCL/1 $1 / 2-5$ allows 
to evaluate children's problems according to 7 empirically based syndrome scales, namely "Emotionally Reactive", "Anxious/Depressed", "Somatic Complaints", "Withdrawn", "Sleep Problems", "Attention Problems", "Aggressive Behaviors", three broad band scales, namely Internalizing, encompassing the first four scales (i.e. "Emotionally Reactive", "Anxious/Depressed", "Somatic Complaints", "Withdrawn"), Externalizing, encompassing the last two scales (i.e. "Attention Problems", "Aggressive Behaviors") and Total scale, as well as to DSM-oriented scales. To limit the number of comparisons, only the 7 empirically based syndrome scales were employed as they cover a wide range of psychopathology. The Italian version showed good psychometric properties [34].

\section{Statistical analyses}

Following Essex et al. [19], Intensity of maternal mood was calculated as the average of the EPDS and STAI-S standardized scores, while Directionality was computed as half difference of the EPDS and STAI-S standardized scores, with a positive score indicating a preponderance of anxiety over depressive symptoms. Intensity and Directionality are uncorrelated and, in contrast to STAI-S and EPDS scores, can be included together in models without multicollinearity issues.

Pearson bivariate correlations and univariate analysis of variance (ANOVA) were preliminarily performed to investigate potential socio-demographic and COVID-related factors (i.e. gender, parity, maternal age, parents education, socio-economic status, parents' employment, financial strains related to the pandemic, home vs office working, number of cohabiting people during the lockdown) that might influence the trajectory of children's emotional and behavioural symptoms and possibly confound the association with maternal mood during the pandemic. Furthermore, as maternal mood before the lockdown might confound the association between maternal mood during the lockdown and children's trajectory of behavioural problems, supplementary analyses were performed including directionality and intensity of maternal mood symptoms at P1 and P2 waves and are reported in Supplementary Materials.

Hierarchical Linear Models (HLMs) were employed to investigate the influence of maternal mood (i.e. intensity and directionality) on the trajectories of children's behavioural and emotional problems, while accounting for the hierarchical structure of the data (three time-points nested within individuals) [35]. HLMs were specified at two levels where subjects were level 2 and time was level 1. Before fitting explanatory models including level-2 predictors, unconditional growth models were run to examine age-related trajectories of children behavioural problems, including both linear and quadratic slopes for time. A random intercept and a random linear slope were included to allow between-persons variability. The models were then expanded by adding between-persons explanatory variables centered around the grand mean and a cross-level interaction with this variable and the within-person predictor time. These models allow us to examine the influence of maternal mood on the agerelated trajectory of child behaviours. The model fit was tested with likelihood deviance difference tests for nested models. HLMs allow to partition different sources of variance and provide robust estimates, despite the presence of missing values, by maximizing all valid data points [36].

Statistical analyses were performed using SPSS 24 and MLWiN 3.05.

\section{Results}

\section{Descriptive analyses and confounders}

The sociodemographic characteristics of mothers and children in the three waves are shown in Table 1. No mothers were affected by the COVID-19 virus, whereas in a few cases $(N=5)$ a family member was. Only 4 mothers reported the loss of a family member or a loved person. However, although this longitudinal sample is composed of participants belonging to a medium-high socio-economic level, the outbreak of the pandemic has represented an economic damage for $13(17 \%)$ of the mothers and $21(29 \%)$ of the fathers. Only 8 mothers $(10.7 \%)$ and 21 fathers $(28.8 \%)$ reported working outside home during the lockdown, whereas the remaining parents were working from home $(42.7 \%$ of mothers and $21.9 \%$ of fathers), on lay-off (12\% of mothers and $24.7 \%$ of fathers) or not working (34.6\% of mothers and $24.6 \%$ of fathers). The mean number of people cohabiting with the child during the lockdown was 3.63 ( $\mathrm{SD}=1.09$ ).

Further, the COVID-19 confinement measures were perceived as having a negative impact on family routine and on the relationship with the child by 36 (48\%) and 23 (31\%) mothers, respectively.

Means and standard deviations of the CBCL/1 1/2-5 syndromes scales in each assessment wave are presented in Table 2. Pairwise bivariate correlations between CBCL 1 $1 / 2-5$ scales scores and Intensity and Directionality scores of maternal mood symptoms in each assessment wave are presented in Table 3.

Preliminary ANOVA showed an effect of gender on children's emotional and behavioural problems. Specifically, males displayed significantly higher levels of Attention Problems, Aggressive Behaviors at both $\mathrm{P} 2$ and L waves and greater Withdrawal problems at $\mathrm{L}$ wave, as compared to females ( $p$ s range 0.00-0.04). Thus, child gender was included as covariate in the main analyses. All other sociodemographic and COVID-related factors were not significantly 
Table 1 Sociodemographic characteristics of the sample

\begin{tabular}{|c|c|c|c|c|c|c|}
\hline & \multicolumn{2}{|c|}{ Wave P1 } & \multicolumn{2}{|c|}{ Wave P2 } & \multicolumn{2}{|c|}{ Wave L } \\
\hline & M & SD & M & SD & M & SD \\
\hline Maternal age & 34.37 & 3.79 & 36.37 & 3.62 & 37.17 & 3.51 \\
\hline \multirow[t]{2}{*}{ Paternal age } & 37.01 & 5.01 & 38.67 & 7.85 & 40.04 & 5.62 \\
\hline & $\mathrm{N}$ & $\%$ & $\mathrm{~N}$ & $\%$ & $\mathrm{~N}$ & $\%$ \\
\hline \multicolumn{7}{|l|}{ Maternal education } \\
\hline Less than 10 years & 9 & 9.6 & 7 & 8.0 & 4 & 6.8 \\
\hline More than 10 years & 85 & 90.4 & 81 & 92.0 & 55 & 93.2 \\
\hline \multicolumn{7}{|l|}{ Paternal education } \\
\hline Less than 10 years & 24 & 25.5 & 18 & 20.5 & 13 & 22.03 \\
\hline More than 10 years & 70 & 74.5 & 70 & 79.5 & 46 & 77.97 \\
\hline \multicolumn{7}{|l|}{ Family SES* } \\
\hline Low & 4 & 4.3 & 3 & 3.4 & 2 & 3.4 \\
\hline Middle & 40 & 42.6 & 34 & 38.6 & 26 & 44.1 \\
\hline High & 50 & 53.2 & 43 & 48.9 & 30 & 50.8 \\
\hline
\end{tabular}

*Percentages for family SES do not add to $100 \%$ due to missing values

Table 2 Means and SDs of the CBCL $1 \frac{1 / 2-5}{2}$ syndrome scales at each wave

\begin{tabular}{|c|c|c|c|c|c|c|c|c|c|}
\hline \multirow{3}{*}{$\begin{array}{l}\text { CBCL/1 } \\
1 / 2-5\end{array}$} & \multicolumn{3}{|l|}{$\mathrm{P} 1$} & \multicolumn{3}{|l|}{$\mathrm{P} 2$} & \multicolumn{3}{|l|}{$\mathrm{L}$} \\
\hline & $\begin{array}{l}\text { Male } \\
(N=50)\end{array}$ & $\begin{array}{l}\text { Female } \\
(N=44)\end{array}$ & $\begin{array}{l}\text { Total } \\
(N=94)\end{array}$ & $\begin{array}{l}\text { Male } \\
(N=47)\end{array}$ & $\begin{array}{l}\text { Female } \\
(N=41)\end{array}$ & $\begin{array}{l}\text { Total } \\
(N=88)\end{array}$ & $\begin{array}{l}\text { Male } \\
(N=32)\end{array}$ & $\begin{array}{l}\text { Female } \\
(N=27)\end{array}$ & Total $(N=59)$ \\
\hline & M (SD) & M (SD) & M (SD) & M (SD) & M (SD) & M (SD) & M (SD) & M (SD) & M (SD) \\
\hline $\begin{array}{l}\text { Emotionally } \\
\text { reactive }\end{array}$ & $1.80(1.51)$ & $1.80(1.95)$ & $1.80(1.72)$ & $2.00(1.55)$ & 1.78 (1.93) & $1.90(1.73)$ & $2.50(2.05)$ & $2.63(1.60)$ & $2.56(1.84)$ \\
\hline $\begin{array}{l}\text { Anxious/ } \\
\text { depressed }\end{array}$ & $1.90(1.51)$ & $1.75(1.70)$ & $1.83(1.60)$ & $2.19(1.61)$ & $1.54(1.85)$ & $1.89(1.74)$ & $3.28(2.36)$ & 2.33 (1.96) & $2.85(2.22)$ \\
\hline $\begin{array}{l}\text { Somatic } \\
\text { complaints }\end{array}$ & $1.00(1.23)$ & $0.95(1.36)$ & $0.98(1.29)$ & $1.17(1.45)$ & $0.83(1.02)$ & $1.01(1.27)$ & $1.31(1.47)$ & $0.89(0.97)$ & $1.12(1.27)$ \\
\hline Withdrawn & $0.74(0.90)$ & $0.59(0.84)$ & $0.67(0.87)$ & $1.21(1.21)$ & $1.10(1.18)$ & $1.16(1.19)$ & $2.34(1.86)$ & $1.41(1.45)$ & $1.91(1.73)$ \\
\hline $\begin{array}{l}\text { Sleep prob- } \\
\text { lems }\end{array}$ & $3.72(2.47)$ & $3.39(2.45)$ & $3.56(2.45)$ & $2.70(2.36)$ & $2.39(2.22)$ & $2.57(2.29)$ & $2.25(1.74)$ & $2.74(3.07)$ & $2.47(2.43)$ \\
\hline $\begin{array}{l}\text { Attention } \\
\text { problems }\end{array}$ & 3.24 (1.77) & $2.52(1.95)$ & $2.90(1.88)$ & $2.68(1.71)$ & $1.39(1.43)$ & $2.08(1.70)$ & $3.36(1.68)$ & $1.96(1.70)$ & $2.73(1.82)$ \\
\hline $\begin{array}{l}\text { Aggressive } \\
\text { behaviour }\end{array}$ & $8.32(3.74)$ & $6.93(4.63)$ & $7.67(4.22)$ & $8.47(4.84)$ & $6.27(5.44)$ & $7.44(5.22)$ & $11.19(5.57)$ & $7.70(4.66)$ & 9.59 (5.42) \\
\hline
\end{tabular}

P1: pre-pandemic wave when child was 1 year old

P2: pre-pandemic wave when child was 3 years old

L: lockdown wave when child was 4 years old

associated with children's emotional and behavioural problems and were discarded for subsequent analyses.

\section{Trajectories of emotional and behavioural problems from the pre-lockdown waves to the lockdown wave}

Unconditional age-related trajectories are depicted in Fig. 1 with dotted lines. Anxious/Depressed and Aggressive Behavior showed significant linear (respectively, estimate $=-0.05$, $\mathrm{SE}=0.02, p=0.02$; estimate $=-0.13, \mathrm{SE}=0.06, p=0.02$ ) and quadratic (respectively, estimate $=0.002, \mathrm{SE}=0.001$, $p=0.001 ;$ estimate $=0.002, \mathrm{SE}=0.001, p<0.001)$ change over time, suggesting a curvilinear increase in those behaviours from P1 to L waves. Likewise, Attention Problems significantly changed over time in a linear (estimate $=-0.13, \mathrm{SE}=0.06, p=0.02$ ) and quadratic (estimate $=0.005, \mathrm{SE}=0.002, p=0.001)$ manner. Specifically, the instantaneous decrease in Attention Problems was followed by a slowing of this trajectory from P2 to L. Emotional Reactivity and Withdrawal showed a significant quadratic change (respectively, estimate $=0.001, \mathrm{SE}=0.001$, $p=0.04$; estimate $=0.001, \mathrm{SE}=0.001, p=0.05)$, indicating 
Table 3 Correlations between CBCL/1 $1 / 2-5$ scales scores and intensity and directionality scores of maternal mood at each assessment wave

\begin{tabular}{|c|c|c|c|c|c|c|}
\hline \multirow[t]{2}{*}{ CBCL/1 1/2-5 } & \multicolumn{2}{|l|}{ P1 } & \multicolumn{2}{|l|}{ P2 } & \multicolumn{2}{|l|}{$\mathrm{L}$} \\
\hline & Intensity & Directionality & Intensity & Directionality & Intensity & Directionality \\
\hline Emotionally reactive & $0.34 * *$ & -0.002 & 0.19 & $-0.24 *$ & $0.49 * *$ & 0.05 \\
\hline Anxious/depressed & $0.31 * *$ & 0.01 & 0.12 & 0.03 & $0.56 * *$ & 0.11 \\
\hline Somatic complaints & 0.19 & 0.07 & 0.15 & -0.11 & $0.35 * *$ & 0.11 \\
\hline Withdrawn & $0.25^{*}$ & -0.16 & -0.003 & 0.05 & $0.50 * * *$ & 0.17 \\
\hline Sleep problems & $0.23^{*}$ & 0.09 & $0.28 * *$ & -0.03 & 0.22 & -0.06 \\
\hline Attention problems & $0.31 * *$ & -0.18 & 0.17 & -0.17 & $0.29 *$ & 0.14 \\
\hline Aggressive behaviour & $0.26^{*}$ & 0.03 & 0.09 & -0.10 & $0.43 * * *$ & -0.19 \\
\hline
\end{tabular}

P1: pre-pandemic wave when child was 1 year old

P2: pre-pandemic wave when child was 3 years old

L: lockdown wave when child was 4 years old

${ }^{*} p<0.05 ; * * p<0.01 ; * * * p<0.001$ a significant curvilinear increase of these problems, whereas Sleep Problems significantly changed over time showing a linear decrease (estimate $=-0.06, \mathrm{SE}=0.03, p=0.02$, quadratic slope not significant $p=0.27$ ) from $\mathrm{P} 1$ to $\mathrm{L}$ waves. Last, Somatic Complaints did not significantly change over time (both linear and quadratic slopes $p \mathrm{~s}>0.69$ ). All unconditional growth models were adjusted for child's gender.

\section{Exposure to maternal mood and children's trajectories of emotional and behavioural problems}

Conditional growth models for the effects of intensity of maternal mood on children's trajectory of emotional and behavioural problems are reported in Table 4. As shown in Fig. 1, the intensity of maternal mood was significantly associated with the linear and quadratic change in Emotional Reactive and Anxious/Depressed over time, while adjusting for child's gender. Specifically, simple slope analysis indicated a curvilinear growth in these problems from P1 to $\mathrm{L}$ waves (for both linear and quadratic slopes $p \mathrm{~s}<0.01$ ) in children of more anxious/depressed mothers. In contrast, the linear and quadratic slopes were not significant in children of less anxious/depressed mothers, indicating no significant changes over time in these problems $(p s>0.31)$. For all these scales, the inclusion of intensity of maternal mood resulted in a significant improvement of the model fit over the unconditional growth models (respectively, for Emotional Reactive deviance difference $(3)=245.99, p<0.001$, for Anxious/Depressed deviance difference $(3)=240.59$, $p<0.001)$. Intensity of maternal mood was also significantly associated with the quadratic slope of children's trajectory of Withdrawn and Aggressive Behavior, while adjusting for child's gender (for Withdrawn deviance difference (3) $=213.25, p<0.001$, for Aggressive Behavior deviance difference $(3)=323.43, p<0.001)$. In particular, a greater increase in Withdrawn or Aggressive Behavior was observed in children of more anxious/depressed mothers
( $p<0.001$ ), while no significant change in children of less anxious/depressed mothers $(p>0.70)$ emerged. In contrast, intensity of maternal mood was not significantly associated with children changes in Somatic Complaints, Sleep and Attention Problems over time, while adjusting for child's gender $(p s>0.05)$.

Last, directionality of maternal mood was not significantly associated with children's change in emotional and behavioural problems over time ( $p s>0.05$ ), while controlling for child's gender.

Controlling for intensity and directionality of maternal mood at 1 and 3 years of age did not substantially change the direction and significance of the results (see Supplementary Table S4).

\section{Discussion}

This study investigated the impact of the COVID-19 lockdown on adaptive behaviours of preschool children, comparing their functioning during the lockdown to that preceding the emergency, and taking also into account the role played by maternal mood in moderating the trajectory of emotional and behavioural problems.

Despite the fact that the first pandemic wave did not severely affect health and economic conditions of our participants in the short term, children's emotional and behavioural problems, especially the internalizing ones, significantly increased from pre- to during the lockdown, taking also into account the effect of child's gender. Specifically, children experienced not only more emotional reactive, anxious-depressive, and withdrawn symptoms but also aggressive symptoms during the lockdown than before. Our data extend previous findings from cross-sectional studies conducted in Italy and abroad in both preschool $[12,18]$ and school age children $[8,12]$ by including a longitudinal assessment of maternal and children's wellbeing before 
Fig. 1 Unconditional growth trajectories for emotional and behavioural problems, while adjusting for gender (dotted lines). Conditional growth trajectories for emotional and behavioural problems in children exposed to lower ($1 \mathrm{SD}$, dashed lines) and higher (+1SD, solid lines) intensity of maternal mood
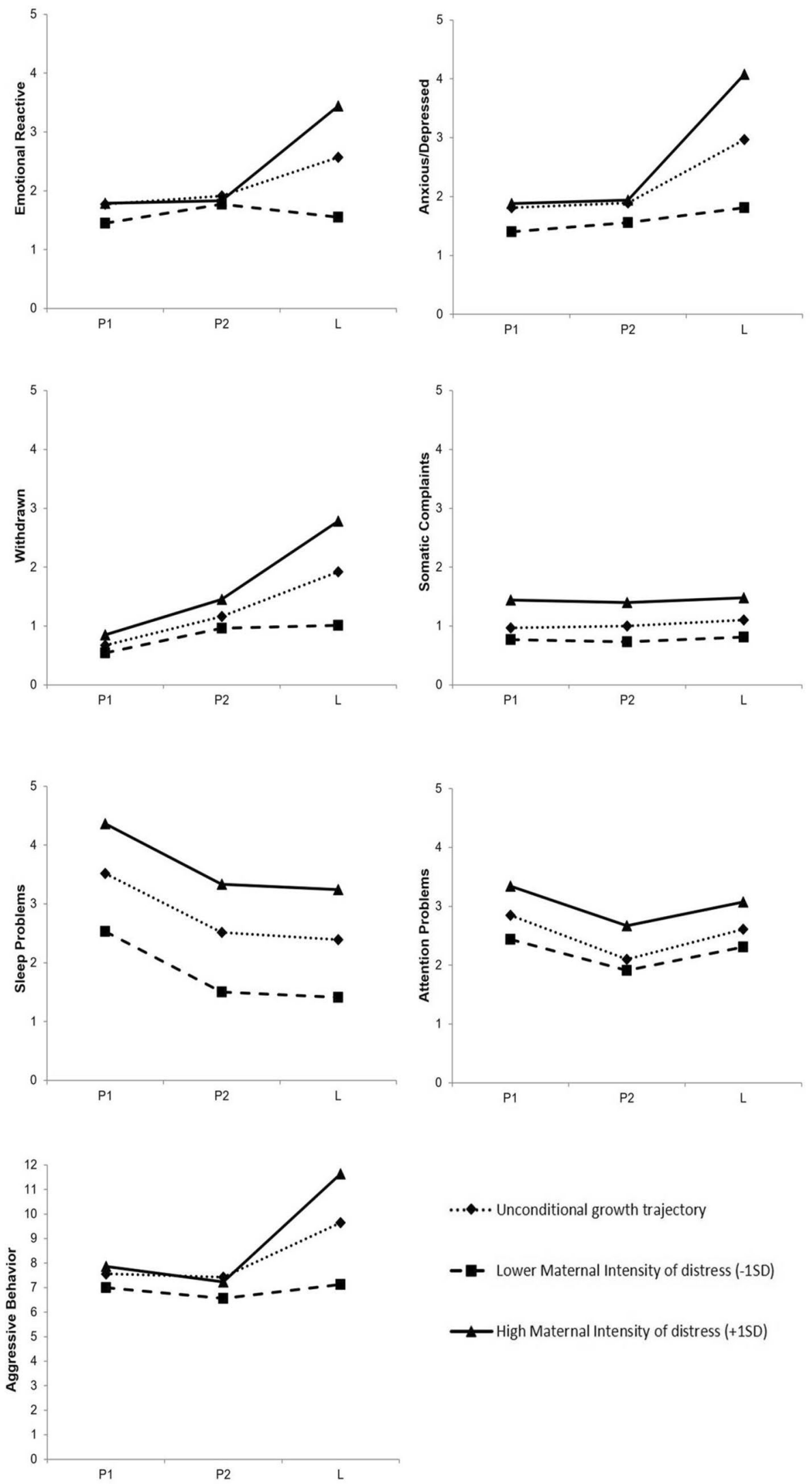

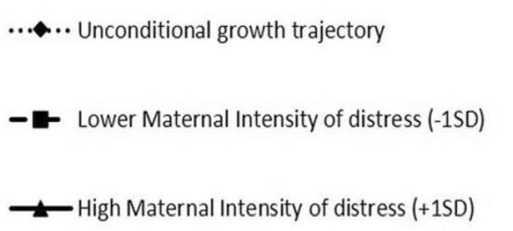




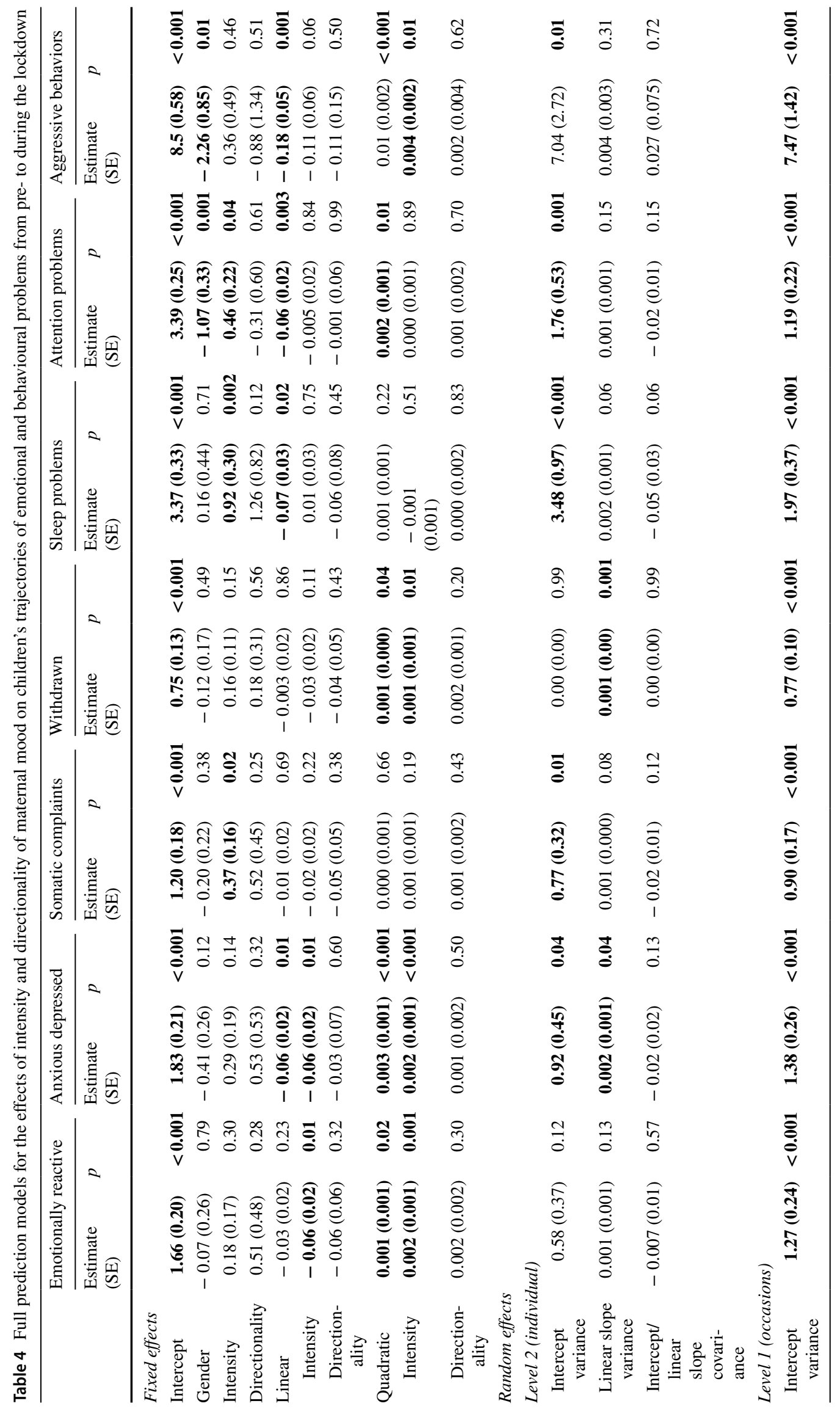


and during the lockdown, thus making more reasonable to attribute the worsening of symptoms to the containment measures. It is also important to note that our study counts on the adoption of two different time points of assessment before the lockdown, which makes the analyses of emotional and behavioural problems trajectory methodologically much more robust. Our results are partly in line with those of Cantiani et al. [22], who found an increase of anxious-depressive and aggressive symptoms in their longitudinal sample of preschool children from pre- to during the lockdown. Similarly, we also found no impact of the COVID-19 lockdown on somatic complaints, sleep and attention problems in our sample, in contrast to results emerging from previous crosssectional studies $[12,37]$. Conversely, we found an intensification of emotional reactive and withdrawn symptoms during the lockdown, thus extending the negative effects of the containment measures to other internalizing syndromes. It is likely that these inconsistencies may be explained by methodological differences related to a more heterogeneous age group, a wide time interval of assessment from pre- to during the lockdown and a different statistical approach in Cantiani et al. study [22]. Taken together, existing data converge to indicate that the COVID-19 lockdown negatively impacted children's psychological well-being, leading to an increase in emotional and behavioural symptoms. We might speculate that children's lack of daily routine, due to prolonged school closures during the lockdown, including a decrease in physical activity and outdoor time, the lack of face-to-face interaction with peers, the increase in screen exposure as well as irregular sleep rhythm [38], might have negatively impacted the children's mental health. However, this hypothesis needs to be specifically addressed in future studies.

More interestingly, our study is among the first to show that the intensity of maternal mood symptoms plays a significant role in determining individual differences in child's emotional and behavioural adjustment from pre- to during the lockdown, independently from the direction (anxiety versus depressive symptoms) of such distress. Particularly, we found that greater maternal mood symptoms were significantly associated with a greater increase in internalizing (i.e. emotional reactive, anxious/depressed, withdrawn) and externalizing (i.e. aggressive) problems in our sample of preschool children during the lockdown. Importantly, the observed associations were found while adjusting for child's gender and remained significant when controlling for the intensity of maternal mood symptoms in the pre-pandemic waves. These findings extend existing evidence on the role of maternal mental health on children's psychological adjustment [39] by showing that maternal psychological distress might be particularly detrimental for children's emotional well-being under stressful circumstances, such as those constituted by the COVID-19 pandemic and subsequent lockdown. This is in line with a "cumulative stress" hypothesis [40] and suggests that the preschoolers' risk to experience emotional and behavioural problems increases as adversity (i.e. lockdown experience and maternal distress) accumulates.

On the contrary, children whose mothers experimented lower levels of mental distress during the lockdown showed no increase in internalizing and externalizing problems from pre- to during the lockdown, thus showing the protective role of maternal mental wellbeing against the effects of the pandemic restrictions on children's emotional and behavioural adjustment.

Insofar as mothers' and children's mental health is intrinsically intertwined, different interrelated pathways might explain the observed associations during the lockdown. It can be speculated that mothers who suffered significant mood symptoms during the COVID-19 lockdown might also have experienced more difficulties in dealing with increased caregiving responsibilities linked to the stay-at-home order. This might have triggered a significant parenting-related exhaustion [15], with consequences on mother-child relationship, which, in turn, might have impacted on the emotional and behavioural adjustment of their children. Likewise, it can also be suggested that the closure of regulated childcare services, due to the lockdown restrictions, might have contributed to exacerbate the impact of maternal psychological wellbeing on children's emotional and behavioural problems, especially the internalizing ones. This hypothesis is supported by previous studies showing that regulated childcare services may have a significant role in buffering the negative effects of maternal depression on internalizing problems in the offspring [41]. Last, as the moderating role of the intensity of maternal distress was evident for almost all internalizing behaviors, investigated through the CBCL $/ 1 \frac{1}{2}-5$, the weight of shared mother-child genetic vulnerability for internalizing symptoms needs to be considered as another possible underlying mechanism.

It is noteworthy that we did not report any significant association between directionality of maternal distress (anxiety versus depressive symptoms) and the trajectory of behavioural problems in preschoolers. This null finding might suggest that maternal depression and anxiety represent a broad risk phenotype for children development, although replication of this finding in clinical samples of depressed and anxious women is needed.

This study should be looked at with some limitations in mind. First, our findings are based on a relatively small lowrisk sample of mother-child dyads, thus soliciting the need of replications with larger and clinical samples. Second, both maternal mood symptoms and children's emotional and behavioural problems were assessed through self-report questionnaires filled in by the mothers, making difficult to exclude possible rater-related bias. Third, the observational 
design of our study does not allow to infer any causality of the association between maternal and children's wellbeing. Last, we only focused on maternal mood symptoms in moderating the trajectory of emotional and behavioural problems from pre- to during the lockdown, without investigating paternal mental wellbeing, which is likely to exert a significant impact on children adjustment [42]. Similarly, we did not take into account the possible moderating role of family social support that remains an open area of inquiry for future research.

Despite these limitations, our study is among the first to evaluate the moderating role of maternal mood on emotional and behavioural problems in young children during the first wave of the COVID-19 in our country, taking also into account both maternal and children functioning before the lockdown. Our findings suggest that maternal emotional well-being in times of emergency might mitigate the adverse impact of the restrictive measures on children's psychological adjustment, thus promoting children's resilience in the face of the pandemic. We are currently re-assessing maternal and children's well-being during the second wave of the COVID-19 in our country, to better understand the acute and persisting effects of the pandemic on preschool children wellbeing.

Supplementary Information The online version contains supplementary material available at https://doi.org/10.1007/s00787-021-01925-0.

Acknowledgements This study was funded by $5 \times 1000$ Funds for biomedical research. Our sincere thanks go to all families who took part in the study.

Funding This study was funded by $5 \times 1000$ Funds for biomedical research.

Data availability The data that support the findings of this study are available from the corresponding author upon reasonable request.

\section{Declarations}

Conflict of interest The authors have no conflict of interest to declare.

\section{References}

1. Vindegaard N, Benros ME (2020) COVID-19 pandemic and mental health consequences: systematic review of the current evidence. Brain Behav Immun 89:531-542. https://doi.org/10. 1016/j.bbi.2020.05.048

2. Racine N, Korczak DJ, Madigan S (2020) Evidence suggests children are being left behind in COVID-19 mental health research. Eur Child Adolesc Psychiatry. https://doi.org/10.1007/ s00787-020-01672-8

3. Cooper K (2020) Don't let children be the hidden victims of COVID-19 pandemic. https://www.unicef.org/press-releases/ dont-let-children-be-hiddenvictimscovid-19-pandemic
4. Bhatia A, Fabbri C, Cerna-Turoff I et al (2020) COVID-19 response measures and violence against children. Bull World Health Organ 98:583. https://doi.org/10.2471/BLT.20.263467

5. UNICEF (2020) UNICEF. UN News. United Nations, 2020. Policy brief: the impact of COVID-19 on children. Policy brief]. United Nations 1-17. https://news.un.org/en/tags/unicef

6. Racine N, Cooke JE, Eirich R et al (2020) Child and adolescent mental illness during COVID-19: a rapid review. Psychiatry Res 292:113307. https://doi.org/10.1016/j.psychres.2020.113307

7. Panda PK, Gupta J, Chowdhury SR et al (2021) Psychological and behavioral impact of lockdown and quarantine measures for COVID-19 pandemic on children, adolescents and caregivers: a systematic review and meta-analysis. J Trop Pediatr 67:1-13. https://doi.org/10.1093/tropej/fmaa122

8. Crescentini C, Feruglio S, Matiz A et al (2020) Stuck outside and inside: an exploratory study on the effects of the COVID-19 outbreak on Italian parents and children's internalizing symptoms. Front Psychol 11:586074. https://doi.org/10.3389/fpsyg.2020. 586074

9. Di Giorgio E, Di Riso D, Mioni G, Cellini N (2020) The interplay between mothers' and children behavioral and psychological factors during COVID-19: an Italian study. Eur Child Adolesc Psychiatry 30:1401-1412. https://doi.org/10.1007/ s00787-020-01631-3

10. Cusinato M, Iannattone S, Spoto A et al (2020) Stress, resilience, and well-being in Italian children and their parents during the COVID-19 pandemic. Int J Environ Res Public Health 17:1-17. https://doi.org/10.3390/ijerph17228297

11. Spinelli M, Lionetti F, Setti A, Fasolo M (2020) Parenting stress during the COVID-19 outbreak: socioeconomic and environmental risk factors and implications for children emotion regulation. Fam Process 60:639-653. https://doi.org/10.1111/famp.12601

12. Viner RM, Russell SJ, Croker $\mathrm{H}$ et al (2020) School closure and management practices during coronavirus outbreaks including COVID-19: a rapid systematic review. Lancet Child Adolesc Health 4:397-404. https://doi.org/10.1016/S2352-4642(20) 30095-X

13. Jiao WY, Wang LN, Liu J et al (2020) Behavioral and emotional disorders in children during the COVID-19 epidemic. J Pediatr 221:264-266. https://doi.org/10.1016/j.jpeds.2020.03.013

14. Romero E, López-Romero L, Domínguez-álvarez B et al (2020) Testing the effects of covid-19 confinement in Spanish children: the role of parents' distress, emotional problems and specific parenting. Int J Environ Res Public Health 17:1-23. https://doi.org/ 10.3390/ijerph17196975

15. Tso WWY, Wong RS, Tung KTS et al (2020) Vulnerability and resilience in children during the COVID-19 pandemic. Eur Child Adolesc Psychiatry 17:1-16. https://doi.org/10.1007/ s00787-020-01680-8

16. Marchetti D, Fontanesi L, Di Giandomenico S et al (2020) The effect of parent psychological distress on child hyperactivity/inattention during the COVID-19 lockdown: testing the mediation of parent verbal hostility and child emotional symptoms. Front Psychol 11:567052. https://doi.org/10.3389/fpsyg.2020.567052

17. Zreik G, Asraf K, Haimov I, Tikotzky L (2021) Maternal perceptions of sleep problems among children and mothers during the coronavirus disease 2019 (COVID-19) pandemic in Israel. J Sleep Res 30:1-7. https://doi.org/10.1111/jsr.13201

18. Murray L, Sinclair D, Cooper P et al (1999) The socioemotional development of 5-year-old children of postnatally depressed mothers. J Child Psychol Psychiatry Allied Discip 40:1259-1271. https://doi.org/10.1017/S002196309900476X

19. Glynn LM, Davis EP, Luby JL et al (2021) A predictable home environment may protect child mental health during the COVID19 pandemic. Neurobiol Stress 14:100291. https://doi.org/10. 1016/j.ynstr.2020.100291 
20. Essex MJ, Kraemer HC, Armstrong JM et al (2006) Exploring risk factors for the emergence of children's mental health problems. Arch Gen Psychiatry 63:1246-1256. https://doi.org/10.1001/archp syc.63.11.1246

21. Shirtcliff EA, Essex MJ (2008) Concurrent and longitudinal associations of basal and diurnal cortisol with mental health symptoms in early adolescence. Dev Psychobiol 50:690-703. https://doi.org/ $10.1038 /$ jid.2014.371

22. Gassman-Pines A (2020) COVID-19 and parent-child psychological well-being. Pediatrics 146:e2020007294. https://doi.org/10. 1542/peds.2020-007294

23. Cantiani C, Dondena C, Riva V et al (2021) Effects of COVID-19 lockdown on the emotional and behavioral profiles of preschool italian children with and without familial risk for neurodevelopmental disorders. Brain Sci 11:477. https://doi.org/10.3390/brain sci11040477

24. Frigerio A, Nazzari S (2021) Antenatal maternal anxiety, maternal sensitivity and toddlers' behavioral problems: an investigation of possible pathways. Early Hum Dev 157:105364. https://doi.org/ 10.1016/j.earlhumdev.2021.105364

25. Nazzari S, Fearon P, Rice F et al (2019) Beyond the HPA-axis: exploring maternal prenatal influences on birth outcomes and stress reactivity. Psychoneuroendocrinology 101:253-262. https:// doi.org/10.1016/j.psyneuen.2018.11.018

26. Nazzari S, Fearon P, Rice F, Ciceri F, Molteni M, Frigerio A (2020) Neuroendocrine and immune markers of maternal stress during pregnancy and infant cognitive development. Dev Psychobiol 62:1100-1110. https://doi.org/10.1002/dev.21967

27. Cox JL, Holden JM, Sagovsky R (1987) Detection of postnatal depression. development of the 10 -item edinburgh postnatal depression scale detection of postnatal depression development of the 10-item edinburgh postnatal depression scale. Br J Psychiatry 150:782-786. https://doi.org/10.1192/bjp.150.6.782

28. Spielberger CD, Gorsuch RL, Lushene RE, et al (1970) State-trait anxiety inventory. Palo Alto

29. Achenbach TM, Rescorla LA (2000) Manual for the ASEBA preschool forms and profiles. University of Vermont, Research Center for Children, Youth \& Families, Burlington

30. Harris PA, Taylor R, Thielke R et al (2009) Research electronic data capture (REDCap) — a metadata-driven methodology and workflow process for providing translational research informatics support. J Biomed Inform 42:377-381

31. Hollingshead AB (1975) Four factor index of social status. Unpublished manuscript, Yale University, Department of Sociology, New Haven.
32. Spielberger CD, Pedrabissi L, Santinello M (2012) STAI state-trait anxiety inventory forma Y: Manuale. Giunti OS Organizzazioni speciali

33. Benvenuti P, Ferrara M, Niccolai C et al (1999) The Edinburgh Postnatal Depression Scale: validation for an Italian sample. J Affect Disord 53:137-141. https://doi.org/10.1016/S01650327(98)00102-5

34. Frigerio A, Cozzi P, Pastore V et al (2006) La valutazione dei problemi emotivo comportamentali in un campione italiano di bambini in eta prescolare attraverso la Child Behavior Checklist e il Caregiver Teacher Report Form. Infanz Adolesc 5:24-37

35. Singer JD, Willett JB (2003) Applied longitudinal data analysis: modeling change and event occurrence. Oxford University Press, New York

36. Hruschka DJ, Kohrt BA, Worthman CM (2005) Estimating between- and within-individual variation in cortisol levels using multilevel models. Psychoneuroendocrinology 30:698-714. https://doi.org/10.1016/j.psyneuen.2005.03.002

37. Orgilés M, Morales A, Delvecchio E et al (2020) Immediate psychological effects of the COVID-19 quarantine in youth from Italy and Spain. Front Psychol 11:1-10. https://doi.org/10.3389/fpsyg. 2020.579038

38. Lee J (2020) Mental health effects of school closures during COVID-19. Lancet Child Adolesc Health 4:421. https://doi.org/ 10.1016/S2352-4642(20)30109-7

39. Goodman SH, Rouse MH, Connell AM et al (2011) Maternal depression and child psychopathology: a meta-analytic review. Clin Child Fam Psychol Rev 14:1-27. https://doi.org/10.1007/ s10567-010-0080-1

40. Nederhof E, Schmidt MV (2012) Physiology and behavior mismatch or cumulative stress: toward an integrated hypothesis of programming effects. Physiol Behav 106:691-700. https://doi.org/ 10.1016/j.physbeh.2011.12.008

41. Herba CM, Tremblay RE, Boivin M et al (2013) Maternal depressive symptoms and children's emotional problems: can early child care help children of depressed mothers? JAMA Psychiatr 70:830-838. https://doi.org/10.1001/jamapsychiatry.2013.1361

42. Ramchandani P, Psychogiou L (2009) Paternal psychiatric disorders and children's psychosocial development. Lancet 374:646653. https://doi.org/10.1016/S0140-6736(09)60238-5 